\title{
Ribbons of superparamagnetic colloids in magnetic field
}

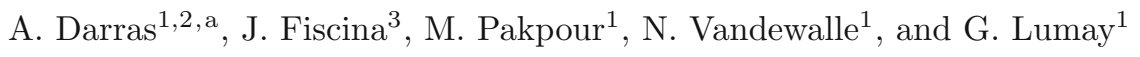 \\ 1 GRASP - Physics Department, University of Liège, B-4000 Liège, Belgium \\ 2 F.R.S.-FRNS, B-1000 Bruxelles, Belgium \\ 3 Experimental Physics, Saarland University, D-66123 Saarbrücken, Germany
}

Received 21 December 2015 and Received in final form 26 February 2016 Published online: 27 April 2016 - (c) EDP Sciences / Società Italiana di Fisica / Springer-Verlag 2016

\begin{abstract}
While the aggregation process of superparamagnetic colloids in strong magnetic field is well known on short time since a few decades, recent theoretical works predicted an equilibrium state reached after a long time. In the present paper, we present experimental observations of this equilibrium state with a two-dimensional system and we compare our data with the predictions of a pre-existing model. Above a critical aggregation size, a deviation between the model and the experimental data is observed. This deviation is explained by the formation of ribbon-shaped aggregates. The ribbons are formed due to lateral aggregation of chains. An estimation of the magnetic energy for chains and ribbons shows that ribbons are stable structures when the number of magnetic grains is higher than $N=30$.
\end{abstract}

\section{Introduction}

Superparamagnetic colloids are magnetic nanoparticles inserted in a matrix of non-magnetic material (polystyrene or silica) to obtain particles with diameter $d$ ranging from $100 \mathrm{~nm}$ to a few micrometers. These composite particles are combining a quasi-zero remanent magnetization and a high magnetic response [1-3]. In applications, the superparamagnetic colloids are functionalized to capture specific targets. After the capture, an inhomogeneous external magnetic field is applied to separate the superparamagnetic particles by magnetophoresis [4]. Moreover, the formation of chains along the magnetic field enhances the separation process. This technique is used for protein isolation, cell separation, waste capture, bacteria processing, chromatography, etc. [1,5-14]. More complex structures of superparamagnetic colloids can be obtained by using rotating fields, even possibly leading to microswimmers or tracers of local dynamics [15-25]. Those complex structures open ways to new kinds of applications as they have unique optical properties and offer tunable structures able to adapt to their environment and execute fuctional tasks $[17,18,21]$. However, the previous studies about those complex structures focus on the properties of the structures obtained, without having a deep understanding of their formation process. The only system for which some model of growth has been published in the literature is the colloidal chains formed under constant magnetic fields.

In colloidal science, it is well known that particles tend to agglomerate due to van der Waals interactions $[26,27]$.

\footnotetext{
a e-mail: alexis.darras@ulg.ac.be
}

In the present experiments, this agglomeration is prevented by covering the particles with carboxyl charged groups. These charged groups create a short range repulsion between the particles, typically within a range of $10 \mathrm{~nm}$ between the particles $[2,28]$. This ensures the stability of the dispersion. In the following, this electrostatic interaction is considered to define an effective size of the particles for the contact of particles which is $10 \mathrm{~nm}$ wider than the natural size of the particles [1]. However, when an external magnetic field $\boldsymbol{B}$ is applied on the suspension, the superparamagnetic particles acquire a magnetic dipole $\boldsymbol{\mu}=\chi V \boldsymbol{B}$, with the magnetic susceptibility $\chi$ of the particles and their volume $V=\frac{4}{3} \pi R^{3}$, given their radius $R$. The particles then interact with each other through dipolar interactions. The potential energy of magnetic interaction between two identical particles at distance $r$ is therefore given by

$$
U(r, \theta)=\frac{\chi^{2} 4 \pi R^{6} B^{2}}{9 \mu_{0}}\left(\frac{1-3 \cos ^{2} \theta}{r^{3}}\right),
$$

with $\theta$ being the angle between the magnetic field $\boldsymbol{B}$ and the line joining the center of the particles. Two particles then attract when they are aligned with the field $\boldsymbol{B}$, while they repel each other if they are side-by-side. This interaction implies that two particles tend to aggregate in a chain aligned with the magnetic field $\boldsymbol{B}$. Several studies, both experimental and theoretical, have shown that superparamagnetic colloids self-organize into chains under those conditions [10,29-33]. Moreover, this aggregation is reversible meaning that the chains break up if the magnetic field $\boldsymbol{B}$ is suppressed. Experimentally, chains of several particles are typically observed $[29,30,32]$ and the 


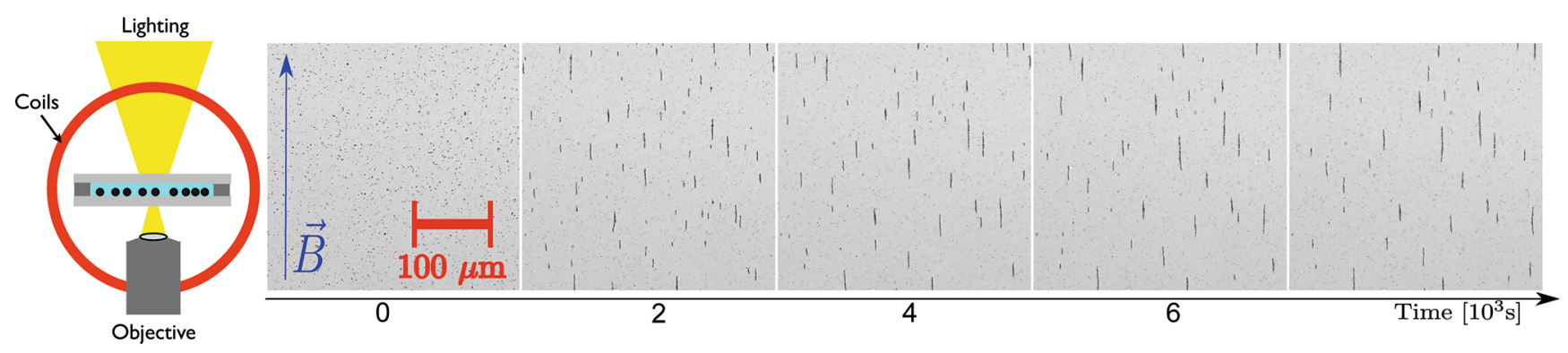

Fig. 1. Left: Sketch of the experimental set-up. A transparent chamber containing the colloidal suspension is placed between two coils generating a constant and homogenous magnetic field $\boldsymbol{B}$. The sample is enlightened from the top and observed from the bottom thanks to an inverted microscope. Right: Evolution of the chains formation along time as observed from one of our experiments. The pictures are part of images obtained with a magnetic field $B=6 \mathrm{G}$. One can observe the formation of chains aligned with the external magnetic field.

growth is successfully described on short time (typically up to 300 s) by a Smoluchowsky equation, predicting a power law behavior of the mean size of the chains $\langle s\rangle \propto t^{z}$ after a transient behavior [29-33].

Recently, Andreu et al. [9] have predicted that such a system, in three dimensions, should reach a thermodynamic equilibrium on long-time experiments. The installation of this equilibrium implies a saturation of the mean size $\langle s\rangle$ of the chains, expressed as a number of particles. This saturation has been predicted by Andreu et al. both by numerical simulations and a thermodynamic analysis $[1,9]$. The equilibrium results from a competition between the magnetic energy of the particles, which is minimal when all the particles are aggregated, and the entropy of mixing, which is maximized for a mixture of different chains. For three dimensional systems, this competition leads to the expression

$$
\langle s\rangle^{(3 D)}=\sqrt{\phi_{0} \exp (\Gamma-1)},
$$

where $\Gamma=\frac{\chi^{2} \pi R^{3} B^{2}}{9 \mu_{0} k_{B} T}$ is a dimensionless parameter comparing the magnetic energy with the thermal energy and $\phi_{0}$ being the total volume fraction of particles. The particles are assumed to form only linear chains, without taking into account the existence of other stable structures such as ribbons or rings $[2,34,35]$. Moreover, a clear experimental observation of the equilibrium state is still lacking.

In the present paper, we provide both a two-dimensional experimental observation of this equilibrium and a suitable adaptation of the model obtained by Andreu et al. [9]. We show that this model is in good agreement with experimental data under a critical mean chains size and we evidence the existence of this critical value. Above this critical value, the formation of stable ribbon-shaped aggregates is observed.

\section{Experimental set-up}

A sketch of the experimental set-up is presented in fig. 1 . The experiments were performed with superparamagnetic microspheres dispersed in water (Estapor ${ }^{\circledR}$ M1-070/60), with a volumic fraction of $\phi=2 \cdot 10^{-3}$. We measured, by image analysis, a radius of particles $r=0.6 \pm 0.3 \mu \mathrm{m}$ while the mean susceptibility, measured by magnetophoresis $[2,36-38]$, is $\chi=0.09 \pm 0.03$. Those values are consistent with previous characterization of that sample found in the literature $[22,23]$. The suspension is placed inside a cylindrical chamber of diameter $D=5 \mathrm{~mm}$ and thickness $h=50 \mu \mathrm{m}$. The chamber is formed by two parallel glass plates. The first glass plate is covered with a $50 \mu \mathrm{m}$ layer of epoxy at the exemption of a circular region. A suspension droplet of $1 \mu \mathrm{l}$ is placed inside this region. Afterward, the second glass plate is placed on the first one. A small quantity of low viscosity silicon oil is placed on the epoxy to asses the watertightness of the chamber. A constant and homogeneous magnetic field $B$ is applied by sending a constant current in surrounding coils at the beginning of each experiment. The magnetic field produced by those coils has been characterized with a Hall probe and is homogeneous within the precision range of the probe of $2 \%$ around the cell. The current is sent in the coils with a constant intensity by a programmable DC power supply GenH-750W from TDK Lambda, with a precision of $0.01 \mathrm{~A}$. The suspension is observed from the bottom with a $10 \times$ magnification. The microscope used is a inverted microscope Olympus IX73, connected to a 4070M-CL Thorlabs Camera with 2048 by 2048 pixels of 16 Bits depth. The images are recorded with a frame rate of $1 \mathrm{fps}$.

\section{Results}

The time evolution of the system is shown in fig. 1. We measured, as a function of time, the normalized mean size $\langle s\rangle$ of the chains formed by the colloidal particles when the magnetic field is applied. This parameter is obtained through image analysis, by averaging the major axis of ellipses fitted on each chain in the image (at least 2000). For short time experiments, after a transient behavior, we obtained a power law growth, as observed in previous studies [29,30,32] (see fig. 2).

For long-time experiments, a saturation of the mean size $\langle s\rangle$ is observed as expected from the theoretical development of Andreu et al. (see fig. 2). When the saturation is reached, we observed some typical behaviors from dynamical equilibria. For example, some parts of the 


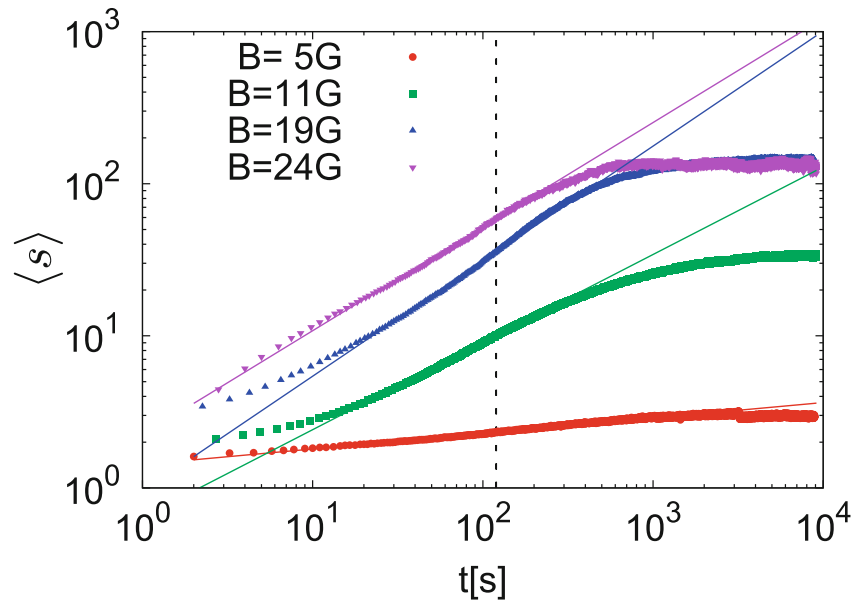

Fig. 2. Log-log plot of the evolution of the mean size of the chains $\langle s\rangle$ during experiments. The mean chains length is expressed in mean diameter of particles. On short time, before the dashed line, a power law growth of the mean size of the chains is obtained after a transient behavior, as observed previously $[29,30,32]$. The solid lines are the fit of a power law on the data before the dashed line (the values of the corresponding exponents are respectively $0.1,0.57,0.75$ and 0.68 with a statistical error of 0.01). However, a saturation of this length is systematically observed on long time, for all the experimental conditions we used. The mean length of the chains at the saturation depends on the magnetic field amplitude. The error is given by the size of the points.

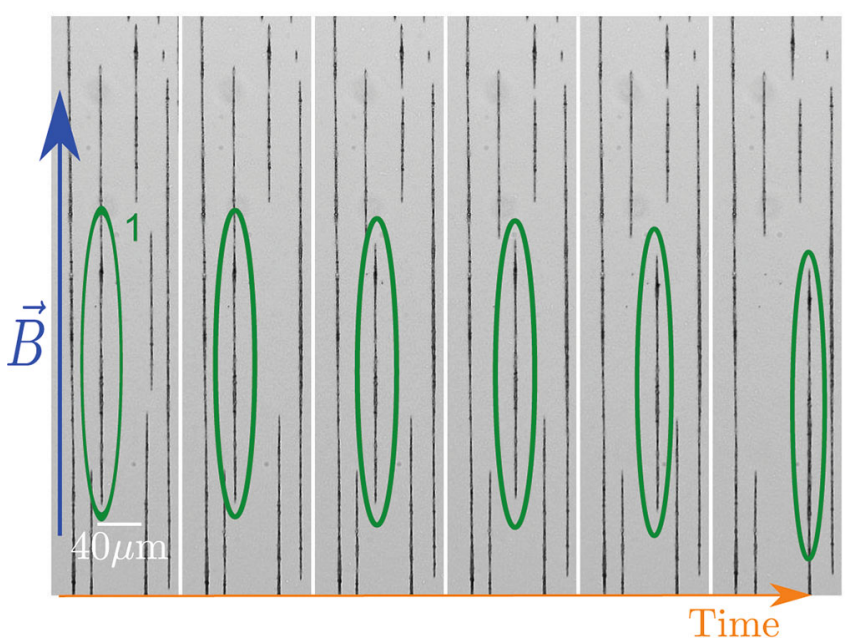

Fig. 3. Images from experiment performed with a magnetic field $B=24 \mathrm{G}$. A part of a chain exhibits a typical behavior from dynamical equilibrium. The part of chain numbered 1 , and circled in every image, leaves the chains where it was first before merging with another one. The time interval between each image is 45 minutes.

longest chains spontaneously leave those chains to merge with some other ones a few minutes later as illustrated in fig. 3. The observation of such behavior confirms that some thermodynamical equilibrium has been reached by the system and indicates that it is not useful to wait a longer time. Such phenomena also implies that the aggre-

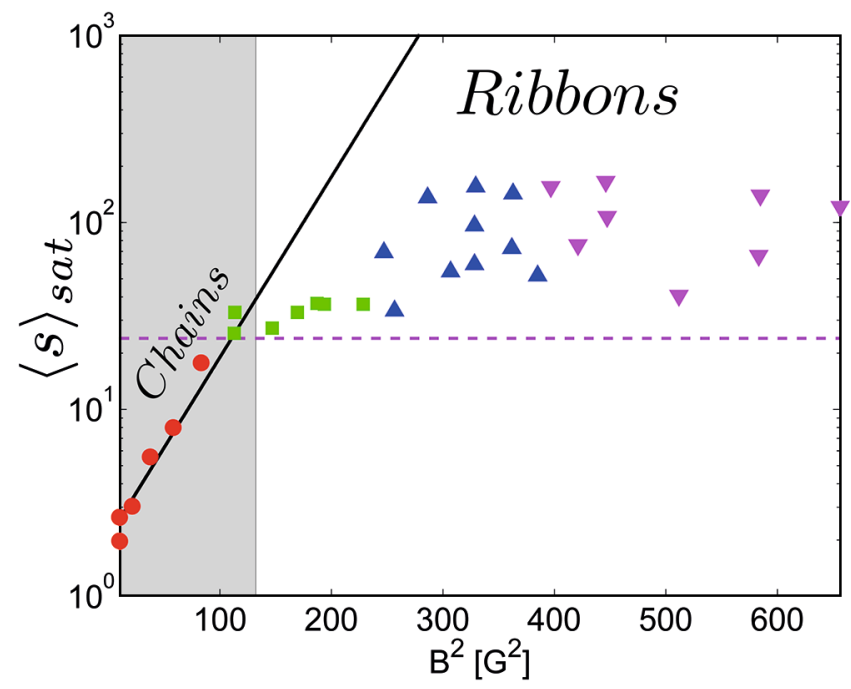

Fig. 4. Mean size of the chains at the saturation $\langle s\rangle_{\text {sat }}$ for different values of the magnetic field. Those measurements have been obtained by considering the lengths of the chains at the plateau in fig. 2 for different experiments where the magnetic field is constant. The measurements are represented by the circles, the squares, the upward and downward triangles, according to the range of the magnetic field. The measurements represented in fig. 2 are then typical behaviors of curves whose saturation lengths are given here with the same symbol. Error bars are smaller than the points. The scaling has been chosen such that the fit of the model $\langle s\rangle=C_{2} \sqrt{\exp \left(C_{1} B^{2}\right)}$ (line) (see sect. 4), calculated with the data in the gray area, draws a straight line. The model predicts higher values than observed ones for strong magnetic fields. We can conclude that the grey area is the range of magnetic field for which the behavior of the chains is sufficient to explain the global mean length of the aggregates, while the white area requires to take into account the existence of the ribbons. The horizontal dashed line is the line $\langle s\rangle=23$, which is an approximation of the limit above which magnetic ribbons wider than one particle have significant influence on the mean size of the aggregates as explained in the text (see sect. 4).

gation of the particles is not completely irreversible, as assumed to establish the Smoluchowsky equation [29-33].

The mean chains size at the saturation $\langle s\rangle_{\text {sat }}$ has been measured for different values of the magnetic field by considering the lengths of the chains at the plateau in fig. 2 for different experiments where the magnetic field is constant (see fig. 4). This maximum size $\langle s\rangle_{\text {sat }}$ first increases with the magnetic field and can be adjusted with an adaptation of the model obtained by Andreu et al. only under a critical value (see sect. 4). After that critical value, the maximum size $\langle s\rangle_{\text {sat }}$ is very sensitive to external noise but is systematically smaller that predicted by such a model.

\section{Discussion}

When the particles are restricted to a two-dimensional plane (as considered for some previous experiments [30]), the model proposed by Andreu et al. for the three dimensional cases should be adapted. Indeed, we have to 
consider the thermal average of the magnetic energy $U(R, \theta)$ of two particles in contact in the range $\theta \in\left[0, \theta_{0}\right]$ implying an attraction between those particles, with $\theta_{0}=$ $\arccos \frac{1}{\sqrt{3}}$. This average involves Jacobians which are different for two dimensional systems. In two dimensions, by using the same approximation as Andreu et al. [9], we obtained a thermal average of $\left\langle\sin ^{2} \theta\right\rangle \approx \frac{1}{3 \Gamma}$ instead of $\frac{2}{3 \Gamma}$ for three-dimensional calculations. In case of a twodimensional situation, the surface fraction $\phi_{0}^{S}$ also replace the volume fraction $\phi_{0}$ and the mean size of the chains at the equilibrium is therefore given by

$$
\langle s\rangle^{(2 D)}=\sqrt{\phi_{0}^{S} \exp \left(\Gamma-\frac{1}{2}\right)} .
$$

With our experimental setup, the situation is more complex that the ideal case presented hereinbefore. Firstly, we measured a dispersion of both radius and susceptibility of the colloidal particles, as described in sect. 2 . Therefore, the estimation of the parameter $\Gamma$ is not straightforward and a fitting parameter $C_{1}$ is used, with $\Gamma=C_{1} B^{2}$. This fitting parameter $C_{1}$ is an effective value of $\frac{\pi R^{3} \chi^{2}}{\mu_{0} 9 k_{B} T}$.

Secondly, our system is not perfectly two dimensional. The particles are confined in a quasi-2D system due to sedimentation induced by gravity. By changing slightly the focal plane height, we observed that the chains and the single particles are not rigorously confined in a plane. The typical height of the confinement region corresponds to twice the particles diameter. This observation is consistent with theoretical comparison of the thermal energy $U_{\mathrm{th}}=$ $k_{B} T$, where $k_{B}$ is the Boltzmann constant and $T$ is the temperature, with the gravitational energy of the particles (taking the buoyancy into account) $U_{g}=\Delta \rho g \frac{4}{3} \pi R^{3} H$, where $H$ is the height of the particles from the bottom of the cell, $R \approx 0.6 \mu \mathrm{m}$ their radius, $g=9.81 \mathrm{~m} / \mathrm{s}^{2}$ the gravitational acceleration and $\Delta \rho \approx 200 \mathrm{~kg} / \mathrm{m}^{3}$ the difference of density between the beads (mainly in polystyrene) and water. Both energy are equals when $H \approx 2.3 \mu \mathrm{m}$, which is then the typical height reached by the particles at the equilibrium and is approximately two diameters of the beads, as observed experimentally.

Then we should have a situation between ideal 2D and 3D cases: $\left\langle\sin ^{2} \theta\right\rangle=\frac{d}{3 \Gamma}$, with $1 \leq d \leq 2$. It is therefore more relevant to express the mean size of the chains as

$$
\langle s\rangle=C_{2} \sqrt{\exp \left(C_{1} B^{2}\right)},
$$

where $C_{2}$ is a second fitting parameter being the effective value of $\sqrt{\phi_{0} \exp (-d)}$. This expression is used to fit the evolution of the mean chain size at the saturation as a function of the magnetic field (see fig. 4). The experimental data can not be fitted with a determination coefficient higher than 0.9 unless the fit is restricted to the points below the critical value $B=12 \mathrm{G}$ giving $\langle s\rangle=24 \pm 3$. Above that value, the measurements give shorter chains than predicted by this fit. The coefficients obtained from

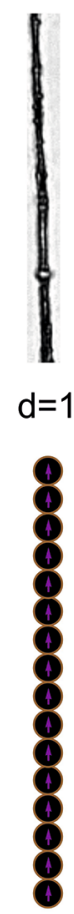

$\mathrm{d}=1$
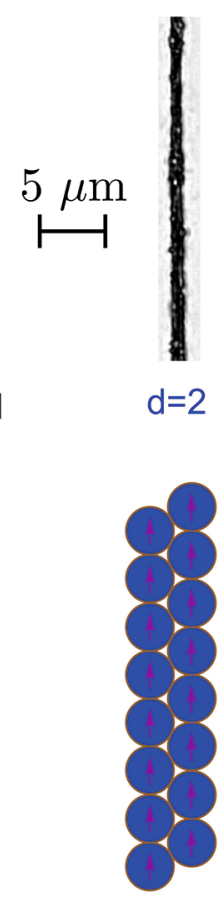

$d=2$
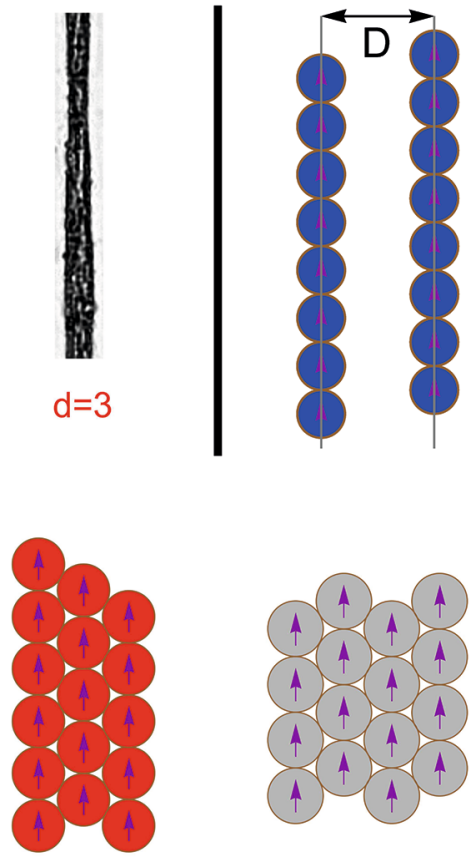

$d=3$

$d=4$
Fig. 5. Structures wider than one particle (magnetic ribbons). Top left: Experimental observations of magnetic ribbons with different widths. Bottom: Structures whose magnetic energy has been numerically computed, from the sum of all the dipoledipole interaction energy. The different structures with 16 particles have been depicted. The small arrow in each circle gives the orientation of the magnetic dipoles. Top right: The distance $D$ used to compute the energy barrier to the formation of the structures is pictured.

the fit presented in fig. 4 are $C_{1}=4.4 \cdot 10^{6} \pm 1.3 \cdot 10^{6} \mathrm{~T}^{-2}$ and $C_{2}=2.1 \pm 1.4$

The fact that chains shorter than predicted by the model are observed is not incompatible with the numerical simulations of Andreu et al. because these simulations implied situations were the mean length of the chains was equal to or below 7 particles [9]. For such situations, the model provides a relevant fit with our data. However, that model assumes that particles always agglomerate in linear chains. This is not always true. Experimentally, we observed lateral aggregation of long chains, leading to structures with more than one particle of width, which are magnetic ribbons, as illustrated in fig. 5 . We then calculated the magnetic energy of different structures, chains and ribbons, illustrated in fig. 5, and assessed the energy barrier to the formation of ribbons by lateral aggregation. To do this we considered a dimensionless mean energy given by

$$
u_{N}(N, d, z)=\frac{1}{N U_{0}} \sum_{i=0}^{N-1} \sum_{j=i+1}^{N} U\left(\left|\boldsymbol{r}_{i}-\boldsymbol{r}_{j}\right|, \theta_{i j}\right),
$$

where $U(r, \theta)$ is defined in eq. (1), $U_{0}=\frac{\chi^{2} \pi R^{3} B^{2}}{18 \mu_{0}}$ is a reference magnetic energy, $\boldsymbol{r}_{i}$ is the position of the particle $i$ of the structure containing $N$ particles, $\theta_{i j}$ is the 


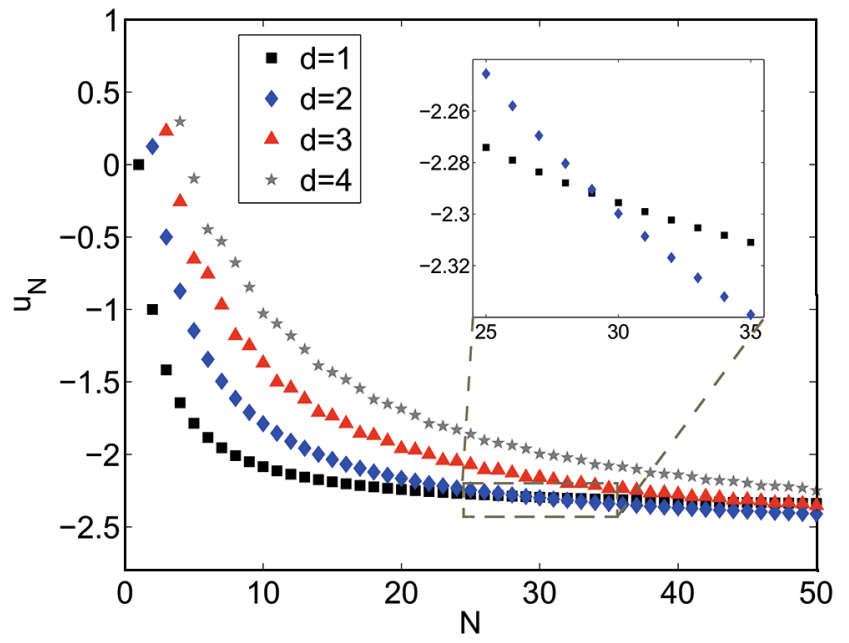

Fig. 6. Mean dimensionless energy of a particle $u_{N}=$ $\frac{1}{N U_{0}} \sum_{i=0}^{N-1} \sum_{j=i+1}^{N} U\left(\left|\boldsymbol{r}_{i}-\boldsymbol{r}_{j}\right|, \theta_{i j}\right)$, with $U_{0}=\frac{\chi^{2} \pi R^{3} B^{2}}{18 \mu_{0}}$, in the different structures as a function of the number of the particles $N$, for $z=0$. The most stable structure with 30 particles is the ribbon with two particles of width. When $N$ increases further, the most stable structure becomes successively the structure with 3 particles $(N \approx 113)$ then 4 particles $(N \approx 263)$.

angle between the direction of the field $\boldsymbol{B}$ and the vector $\boldsymbol{r}_{i}-\boldsymbol{r}_{j}, d$ denotes the type of structure as on fig. 5 and $z \equiv \frac{D-2 R \cos \left(\frac{\pi}{6}\right)}{2 R}$ is a dimensionless distance used to compute the barrier energy to the formation of the ribbons by lateral aggregation. This reasoning is similar to analyses from previous studies published in recent articles [34,35], and the asymptotic value we obtained for $\lim _{N \rightarrow \infty} u_{N}$ in the case of linear chains approaches $-2 \zeta(3) \approx-2.404$ as expected from the results of those studies.

This estimation shows that ribbons composed of two linear chains side by side are the most stable structures for aggregates of 30 particles (see fig. 6). When aggregates contain on average 30 particles, there is a mixture of linear chains of 30 particles and ribbons composed of two linear chains of 15 particles. The mean size of those aggregates is then about 23 particles. This is approximately the size from which the model diverges from our experimental data (see fig. 4).

We also noticed that above the critical value of the field, which seems actually to be determined by a critical size of chains, our measurements were very sensitive to external noise such as residual flows from the fluid. We believe this sensitivity to be due to the energy barrier preventing the appearance of the most stable structures. Indeed, fig. 7 clearly shows there is a barrier of potential to the formation of the structure by lateral aggregation. This implies a complex configuration space with metastable states for the chains. It is worthwhile to notice that the $U_{0}$ reference energy can be expressed as $k_{B} T \frac{C_{1} B^{2}}{2}$, and ribbons have been observed in our cases for $B^{2}$ ranging from $2 \cdot 10^{-6} \mathrm{~T}^{2}$ to $6 \cdot 10^{-6} \mathrm{~T}^{2}$. In such cases, $U_{0}$ ranges from $4.4 k_{B} T$ to $13.2 k_{B} T$. The potential barriers we have computed were ranging from $0.5 U_{0}$ to $2 U_{0}$, depending on the

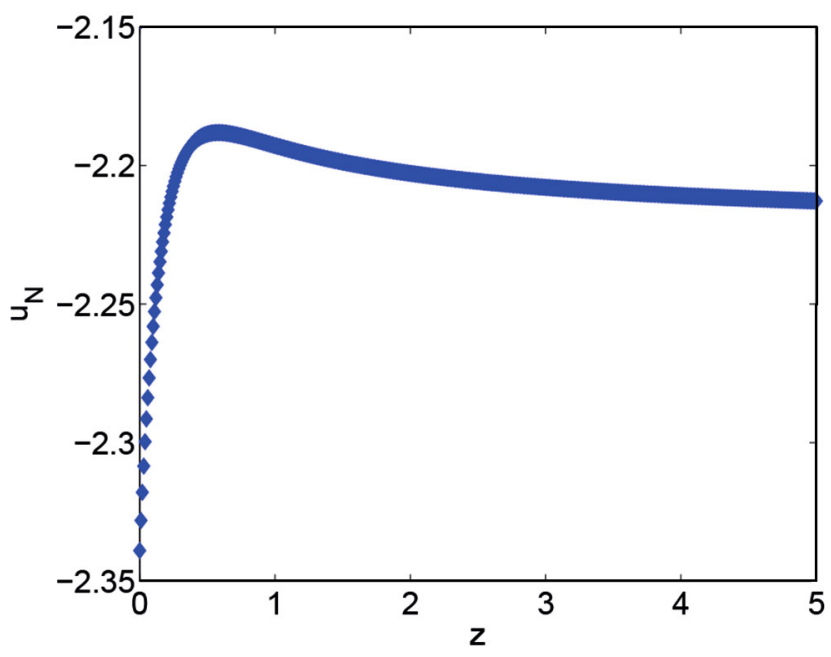

Fig. 7. Dimensionless mean magnetic energy $u_{N}$ for a ribbon with two particles of width and $N=35$ particles. The variable $z \equiv \frac{D-2 R \cos \left(\frac{\pi}{6}\right)}{2 R}$ is a dimensionless distance separating the two chains forming the ribbon, as pictured in the fig. 5. One can distinguish an energy barrier.

number of particles in the considered structures and the width $d$ of the ribbons. Then, as weakest barriers are of the order of $2 k_{B} T$, thermal agitation of the particles itself is likely to trigger some ribbons formation, but not as much as observed in our experiments. Actually, some external noise can move one or several chains from a metastable state to another one and then changing the mean length of the chains. In our experimental setup, such noise can be produced, for instance, by the flow around a small moving air or oil bubble accidentally trapped in the chamber with the colloidal suspension. During our experiments, which can last up to 5 hours, such perturbations are likely to occur, but not systematically.

\section{Conclusion}

In the present paper, we reported the first experimental observation of the saturation predicted by the model of Andreu et al. [9]. We experimentally validated this model on a range of experimental parameters such that $\langle s\rangle<23$, but we showed that this model diverges with experimental data above a critical value for which we proposed an explanation, by highlighting the existence of magnetic ribbons. In the future, the model could be improved by considering the existence of these ribbons, in order to predict the mean size of the chains above the critical size. Our observations also highlighted that the hypothesis of irreversible aggregation assumed by the usual Smoluchowsky equation is not verified for long-time experiments. A more accurate equation taking into account some terms expressing the reversibility of this aggregation could be developed. The physical mechanisms we highlighted here could also be a starting point to develop protocols of controlled ribbons generation. 
A.D. is financially supported by FNRS as research fellow. J.F. thanks the FNRS and Alexander Von Humboldt Foundation. This work was financially supported by the FNRS (Grant PDR T.0043.14) and by the University of Liège (Starting Grant C$13 / 88)$.

\section{References}

1. J. Faraudo, J.S. Andreu, J. Camacho, Soft Matter 9, 6654 (2013).

2. J. Faraudo, J. Camacho, Colloid Polym. Sci. 288, 207 (2010).

3. J.W. Tavacoli, P. Bauër, M. Fermigier, D. Bartolo, J. Heuvingh, O. du Roure, Soft Matter 9, 9103 (2013).

4. C.T. Yavuz, A. Prakash, J. Mayo, V.L. Colvin, Chem. Eng. Sci. 64, 2510 (2009).

5. C.T. Yavuz, J. Mayo, W.Y. William, A. Prakash, J.C. Falkner, S. Yean, L. Cong, H.J. Shipley, A. Kan, M. Tomson et al., Science 314, 964 (2006).

6. K.M. Krishnan, IEEE Trans. Magn. 46, 2523 (2010).

7. J.L. Corchero, A. Villaverde, Trends Biotechnol. 27, 468 (2009).

8. U. Jeong, X. Teng, Y. Wang, H. Yang, Y. Xia, Adv. Mater. 19, 33 (2007).

9. J.S. Andreu, J. Camacho, J. Faraudo, Soft Matter 7, 2336 (2011).

10. G.P. Gajula, M.T. Neves-Petersen, S.B. Petersen, Appl. Phys. Lett. 97, 103103 (2010).

11. K.S. Khalil, A. Sagastegui, Y. Li, M.A. Tahir, J.E. Socolar, B.J. Wiley, B.B. Yellen, Nat. Commun. 3, 794 (2012).

12. Y. Gurevich, Y. Mankov, R. Khlebopros, Dokl. Phys. 11, 478 (2013).

13. F. Martinez-Pedrero, P. Tierno, Phys. Rev. Appl. 3, 051003 (2015) DOI: 10.1103/PhysRevApplied.3.051003.

14. H. Carstensen, V. Kapaklis, M. Wolff, Phys. Rev. E 92, 012303 (2015) DOI: 10.1103/PhysRevE.92.012303.

15. K. Muller, N. Osterman, D. Babic, C.N. Likos, J. Dobnikar, A. Nikoubashman, Langmuir 30, 5088 (2014).

16. M. Llera, J. Codnia, G.A. Jorge, J. Magn. \& Magn. Mater 384, 93 (2015).
17. R.M. Erb, H.S. Son, B. Samanta, V.M. Rotello, B.B. Yellen, Nature 457, 999 (2009).

18. F. Martinez-Pedrero, P. Tierno, Phys. Rev. Appl. 3, 051003 (2015).

19. F. Martinez-Pedrero, A. Ortiz-Ambriz, I. Pagonabarraga, P. Tierno, Phys. Rev. Lett. 115, 138301 (2015).

20. H. Carstensen, V. Kapaklis, M. Wolff, Phys. Rev. E 92, 012303 (2015).

21. P. Liu, J.W. De Folter, A.V. Petukhov, A.P. Philipse, Soft matter 11, 6201 (2015)

22. P. Domínguez-García, J. Pastor, M. Rubio, Eur. Phys. J. E 34, 1 (2011).

23. P. Domínguez-García, S. Melle, J. Pastor, M. Rubio, Phys. Rev. E 76, 051403 (2007).

24. K.V. Edmond, H. Park, M.T. Elsesser, G.L. Hunter, D.J. Pine, E.R. Weeks, Chaos-Woodbury 21, 041103 (2011).

25. R. Dreyfus, J. Baudry, M.L. Roper, M. Fermigier, H.A. Stone, J. Bibette, Nature 437, 862 (2005).

26. D.H. Everett, Basic Principles of Colloid Science (Royal Society of Chemistry, 1988).

27. J.N. Israelachvili, Intermolecular and Surface Forces, revised third edition (Academic Press, 2011).

28. C.P. Royall, W.C. Poon, E.R. Weeks, Soft Matter 9, 17 (2013).

29. J.H. Promislow, A.P. Gast, M. Fermigier, J. Chem. Phys. 102, $5492(1995)$

30. S. Fraden, A.J. Hurd, R.B. Meyer, Phys. Rev. Lett. 63 2373 (1989)

31. M. Kolb, Phys. Rev. Lett. 53, 1653 (1984).

32. F. Martínez-Pedrero, M. Tirado-Miranda, A. Schmitt, J. Callejas-Fernández, Phys. Rev. E 76, 011405 (2007).

33. S. Miyazima, P. Meakin, F. Family, Phys. Rev. A 36, 1421 (1987).

34. R. Messina, L.A. Khalil, I. Stanković, Phys. Rev. E 89, $011202(2014)$

35. N. Vandewalle, S. Dorbolo, New J. Phys. 16, 013050 (2014).

36. M. Suwa, H. Watarai, Anal. Chim. Acta 690, 137 (2011).

37. G. De Las Cuevas, J. Faraudo, J. Camacho, J. Phys. Chem. C 112, 945 (2008).

38. J. Ally, A. Amirfazli, Colloids Surf. A 360, 120 (2010). 